\title{
New Convolutions with Hermite Weight Functions*
}

\author{
L. P. Castro ${ }^{\dagger}$ A. S. Silva $a^{\ddagger}$ N. M. Tuan ${ }^{\S}$ \\ In honor of Professor Roland Duduchava on the occasion of his 75th birthday
}

\begin{abstract}
In this paper, we are working with convolutions on the positive half-line, for Lebesgue integrable functions. Six new convolutions are introduced. Factorization identities for these convolutions are derived, upon the use of Fourier sine and cosine transforms and Hermite functions. Such convolutions allow us to consider systems of convolution type equations on the half-line. Using two different methods, such systems of convolution integral equations will be analyzed. Conditions for their solvability will be considered and, under such conditions, their solutions are obtained.
\end{abstract}

Keywords Convolution (Sine and cosine) integral transforms Hermite functions Factorization property Integral equations of convolution type

Mathematics Subject Classification (2010) 44A35 65R10 33C45 42A85 45E10

\section{Introduction}

Despite all the previous developments in the area of convolution type operators and equations, and their applications (cf., e.g., [6]), several additional investigations in this field are going on in recent years. Namely, new convolutions are continuing to be introduced and applied to a great number of situations with particular emphasis within engineering problems (cf., e.g., $[1,2,3,4,5])$. Anyway, the majority of the introduced new convolutions are being considered in the full real line (or "full space") situation in which the use of the full space facilitates the action of the convolutions (as well as the existence and representation of their inverses). Different context is the "non-full real line" case, in which the action and image of new convolutions need to be again considered in such restricted domains. This also brings additional difficulties related with the existence and representation of the associated inverse transformations.

The last situation is considered in this paper. We need to start by introducing some auxiliary notions and results.

${ }^{*}$ This is a post-peer-review, pre-copyedit version of an article published in Bull. Iran. Math. Soc.. The final authenticated version is available online at: http://dx.doi.org/10.1007/s41980-020-00496-1, https: //link.springer.com/article/10.1007/s41980-020-00496-1.

${ }^{\dagger}$ CIDMA-Center for Research and Development in Mathematics and Applications, University of Aveiro, Portugal, castro@ua.pt

${ }^{\ddagger}$ CIDMA-Center for Research and Development in Mathematics and Applications, University of Aveiro, Portugal, anabela.silva@ua.pt

$\S$ Department of Mathematics, VNU University of Education, Viet Nam National University, Hanoi, Vietnam, nguyentuan@vnu.edu.vn 
Let $\mathbb{R}_{+}:=(0, \infty)$ be the set of positive real numbers and let $L^{1}\left(\mathbb{R}_{+}\right)$be the space of all (Lebesgue) measurable complex-valued functions $f: \mathbb{R}_{+} \rightarrow \mathbb{C}$ with the finite norm:

$$
\|f\|_{L^{1}\left(\mathbb{R}_{+}\right)}:=\int_{0}^{\infty}|f(u)| \mathrm{d} u
$$

For any element $f \in L^{1}\left(\mathbb{R}_{+}\right)$, let us consider the cosine Fourier transform, $T_{c}$, and its inverse defined, respectively, by

$$
\begin{aligned}
\left(T_{c} f\right)(x) & =\sqrt{\frac{2}{\pi}} \int_{0}^{\infty} \cos (x y) f(y) \mathrm{d} y, \quad x \in \mathbb{R}_{+}, \\
f(y) & =\sqrt{\frac{2}{\pi}} \int_{0}^{\infty} \cos (x y)\left(T_{c} f\right)(x) \mathrm{d} x .
\end{aligned}
$$

Similarly, we define the sine Fourier transform, $T_{s}$, and its inverse by

$$
\begin{aligned}
\left(T_{s} f\right)(x) & =\sqrt{\frac{2}{\pi}} \int_{0}^{\infty} \sin (x y) f(y) \mathrm{d} y, \quad x \in \mathbb{R}_{+}, \\
f(y) & =\sqrt{\frac{2}{\pi}} \int_{0}^{\infty} \sin (x y)\left(T_{s} f\right)(x) \mathrm{d} x,
\end{aligned}
$$

respectively.

We recall that associated with the Fourier cosine integral transform, Sneddon [8] introduced, in 1951, a convolution of two elements $f$ and $g$ in the form:

$$
\left(f *^{0} g\right)(x)=\frac{1}{\sqrt{2 \pi}} \int_{0}^{\infty} f(y)[g(|x-y|)+g(x+y)] \mathrm{d} y, \quad x \in \mathbb{R}_{+} .
$$

This convolution enjoys the following important factorization property:

$$
T_{c}\left(f *^{0} g\right)=\left(T_{c} f\right)\left(T_{c} g\right) .
$$

It was also introduced by I. N. Sneddon, a generalized convolution for the Fourier sine and cosine transforms, defined by

$$
\left(f *^{1} g\right)(x)=\frac{1}{\sqrt{2 \pi}} \int_{0}^{\infty} f(y)[g(|x-y|)-g(x+y)] \mathrm{d} y, \quad x \in \mathbb{R}_{+},
$$

and which admits the following factorization property:

$$
T_{s}\left(f *^{1} g\right)=\left(T_{s} f\right)\left(T_{c} g\right) .
$$

In the next section, we introduce new convolutions for the Fourier sine and cosine integral transforms which are related with Hermite functions. We will denote the Hermite functions by $\Phi_{n}$, for $n \in \mathbb{N}_{0}$, which are defined as

$$
\Phi_{n}(x)=(-1)^{n}\left(2^{n} n ! \sqrt{\pi}\right)^{-\frac{1}{2}} e^{\frac{x^{2}}{2}} \frac{d^{n}}{\mathrm{~d} x^{n}} e^{-x^{2}}, \quad x \in \mathbb{R} .
$$

The Fourier transform is an essential tool in many areas of mathematics and science. This transform and its inverse, denoted, respectively, by $\mathcal{F}$ and $\mathcal{F}^{-1}$, are defined by

$$
\begin{aligned}
(\mathcal{F} f)(x) & =\frac{1}{\sqrt{2 \pi}} \int_{-\infty}^{+\infty} e^{-i x y} f(y) \mathrm{d} y \\
{\left[\mathcal{F}^{-1}(\mathcal{F} f)\right](y) } & =f(y)=\frac{1}{\sqrt{2 \pi}} \int_{-\infty}^{+\infty} e^{i x y}(\mathcal{F} f)(x) \mathrm{d} x .
\end{aligned}
$$


The Hermite functions, $\Phi_{n}(x)$, are the eigenfunctions of the Fourier transform associated with the eigenvalues $1,-1, i,-i$. It can be verified by studying the differential equation

$$
\Phi_{n}^{\prime \prime}(x)+\left(2 n+1-x^{2}\right) \Phi_{n}(x)=0
$$

for which $\Phi_{n}(x)$ is a solution (cf. [9]). In what follows, we will use these properties:

$$
\Phi_{n}(x)=(-i)^{n}\left(\mathcal{F}^{-1} \Phi_{n}\right)(x), \quad \text { and } \quad \Phi_{n}(-x)=(-1)^{n} \Phi_{n}(x) .
$$

Moreover, we should observe that $\Phi_{n}$ are absolutely integrable functions

$$
\int_{0}^{\infty}\left|\Phi_{n}(x)\right| \mathrm{d} x<\infty
$$

\section{New Convolutions}

In this section, we will propose six new (classes of) convolutions, associated with Fourier sine and cosine integral transforms, and we will present some of their properties. We start by defining two new convolutions based on Hermite functions and some shift operations.

Definition 1. For any $f$ and $g \in L^{1}\left(\mathbb{R}_{+}\right)$, we define the convolution operators $\circledast^{e}$ and $\circledast^{o}$ by:

$$
\begin{aligned}
\left(f \circledast^{e} g\right)(u) & =\frac{(-1)^{k}}{2 \pi} \int_{0}^{\infty} \int_{0}^{\infty}\left[\Phi_{2 k}(u-y-t)+\Phi_{2 k}(-u-y-t)\right. \\
& \left.+\Phi_{2 k}(u-y+t)+\Phi_{2 k}(-u-y+t)\right] f(y) g(t) \mathrm{d} y \mathrm{~d} t, \\
\left(f \circledast^{o} g\right)(u) & =\frac{(-1)^{k}}{2 \pi} \int_{0}^{\infty} \int_{0}^{\infty}\left[\Phi_{2 k+1}(u-y-t)-\Phi_{2 k+1}(-u-y-t)\right. \\
& \left.+\Phi_{2 k+1}(u-y+t)-\Phi_{2 k+1}(-u-y+t)\right] f(y) g(t) \mathrm{d} y \mathrm{~d} t,
\end{aligned}
$$

$k \in \mathbb{N}_{0}$.

Theorem 1. Let $f, g \in L^{1}\left(\mathbb{R}_{+}\right)$. The convolutions $\left.\circledast\right)^{e}$ and $\circledast^{o}$ of functions $f$ and $g$ belong to $L^{1}\left(\mathbb{R}_{+}\right)$and satisfy the following weighted factorization identities associated with cosine and sine Fourier integral transforms $T_{c}$ and $T_{s}$ :

$$
\begin{aligned}
& T_{c}\left(f \circledast^{e} g\right)(x)=\Phi_{2 k}(x)\left(T_{c} f\right)(x)\left(T_{c} g\right)(x), \\
& T_{s}\left(f \circledast^{o} g\right)(x)=\Phi_{2 k+1}(x)\left(T_{c} f\right)(x)\left(T_{c} g\right)(x), \quad k \in \mathbb{N}_{0} .
\end{aligned}
$$

Proof. Let $f, g \in L^{1}\left(\mathbb{R}_{+}\right)$. We have that:

$$
\begin{aligned}
& \int_{0}^{\infty}\left|\left(f \circledast^{e} g\right)(u)\right| \mathrm{d} u \\
= & \frac{1}{2 \pi} \int_{0}^{\infty} \int_{0}^{\infty} \int_{0}^{\infty} \mid \Phi_{2 k}(u-y-t)+\Phi_{2 k}(-u-y-t) \\
& +\Phi_{2 k}(u-y+t)+\Phi_{2 k}(-u-y+t)|| f(y)|| g(t) \mid \mathrm{d} y \mathrm{~d} t \mathrm{~d} u \\
\leq & \frac{1}{2 \pi} \int_{0}^{\infty}|f(y)| \int_{0}^{\infty}|g(t)|\left[\int_{0}^{\infty}\left|\Phi_{2 k}(u-y-t)\right| \mathrm{d} u\right. \\
& +\int_{0}^{\infty}\left|\Phi_{2 k}(-u-y-t)\right| \mathrm{d} u+\int_{0}^{\infty}\left|\Phi_{2 k}(u-y+t)\right| \mathrm{d} u
\end{aligned}
$$




$$
\left.+\int_{0}^{\infty}\left|\Phi_{2 k}(-u-y+t)\right| \mathrm{d} u\right] \mathrm{d} t \mathrm{~d} y
$$

On the other hand, since $\Phi(-x)=(-1)^{n} \Phi_{n}(x), x \in \mathbb{R}, n \in \mathbb{N}_{0}$, we have that:

$$
\begin{aligned}
& \int_{0}^{\infty}\left|\Phi_{n}(u+\alpha)\right| \mathrm{d} u+\int_{0}^{\infty}\left|\Phi_{n}(-u+\alpha)\right| \mathrm{d} u \\
= & \int_{\alpha}^{\infty}\left|\Phi_{n}(s)\right| \mathrm{d} s+\int_{-\infty}^{\alpha}\left|\Phi_{n}(s)\right| \mathrm{d} s \\
= & \int_{-\infty}^{\infty}\left|\Phi_{n}(s)\right| \mathrm{d} s=2 \int_{0}^{\infty}\left|\Phi_{n}(s)\right| \mathrm{d} s
\end{aligned}
$$

for any $\alpha \in \mathbb{R}$. Thus, we can conclude that:

$$
\int_{0}^{\infty}\left|\left(f \circledast^{e} g\right)(u)\right| \mathrm{d} u \leq \frac{2}{\pi} \int_{0}^{\infty}|f(t)| d t \times \int_{0}^{\infty}|g(t)| d t \times \int_{0}^{\infty}\left|\Phi_{2 k}(t)\right| \mathrm{d} t<\infty .
$$

Thus, $f \circledast{ }^{e} g \in L^{1}\left(\mathbb{R}_{+}\right)$.

In the same way, using (2.3), we have that:

$$
\begin{aligned}
& \int_{0}^{\infty}\left|\left(f \circledast^{o} g\right)(u)\right| \mathrm{d} u \\
= & \frac{1}{2 \pi} \int_{0}^{\infty} \int_{0}^{\infty} \int_{0}^{\infty} \mid \Phi_{2 k+1}(u-y-t)-\Phi_{2 k+1}(-u-y-t) \\
& +\Phi_{2 k+1}(u-y+t)-\Phi_{2 k+1}(-u-y+t)|| f(y)|| g(t) \mid \mathrm{d} y \mathrm{~d} t \mathrm{~d} u \\
\leq & \frac{1}{2 \pi} \int_{0}^{\infty}|f(y)| \int_{0}^{\infty}|g(t)|\left[\int_{0}^{\infty}\left|\Phi_{2 k+1}(u-y-t)\right| \mathrm{d} u\right. \\
& +\int_{0}^{\infty}\left|\Phi_{2 k+1}(-u-y-t)\right| d u+\int_{0}^{\infty}\left|\Phi_{2 k+1}(u-y+t)\right| \mathrm{d} u \\
& \left.+\int_{0}^{\infty}\left|\Phi_{2 k+1}(-u-y+t)\right| d u\right] \mathrm{d} t \mathrm{~d} y \\
\leq & \frac{2}{\pi} \int_{0}^{\infty}|f(t)| d t \times \int_{0}^{\infty}|g(t)| \mathrm{d} t \times \int_{0}^{\infty}\left|\Phi_{2 k+1}(t)\right| \mathrm{d} t<\infty,
\end{aligned}
$$

and we conclude that $f \circledast^{o} g \in L^{1}\left(\mathbb{R}_{+}\right)$.

Let us prove the factorization identities. Using properties (1.1) and the formulas:

$$
\begin{aligned}
2 \cos \alpha \cos \beta & =\cos (\alpha+\beta)+\cos (\alpha-\beta), \quad \alpha, \beta \in \mathbb{R}, \\
2 \cos z & =e^{i z}+e^{-i z}, \quad z \in \mathbb{C},
\end{aligned}
$$

we have:

$$
\begin{aligned}
& \Phi_{n}(x)\left(T_{c} f\right)(x)\left(T_{c} g\right)(x)=(-i)^{n}\left(\mathcal{F}^{-1} \Phi_{n}\right)(x)\left(T_{c} f\right)(x)\left(T_{c} g\right)(x) \\
= & \frac{\sqrt{2}}{\pi \sqrt{\pi}}(-i)^{n} \int_{-\infty}^{\infty} e^{i h x} \Phi_{n}(h) \mathrm{d} h \int_{0}^{\infty} \cos (x y) f(y) \mathrm{d} y \int_{0}^{\infty} \cos (x t) g(t) \mathrm{d} t \\
= & \frac{\sqrt{2}}{\pi \sqrt{\pi}}(-i)^{n}\left[\int_{-\infty}^{0} e^{i h x} \Phi_{n}(h) \mathrm{d} h+\int_{0}^{\infty} e^{i h x} \Phi_{n}(h) \mathrm{d} h\right]
\end{aligned}
$$




$$
\begin{aligned}
& \times \int_{0}^{\infty} \cos (x y) f(y) d y \int_{0}^{\infty} \cos (x t) g(t) \mathrm{d} t \\
= & \frac{\sqrt{2}}{\pi \sqrt{\pi}}(-i)^{n}\left[\int_{0}^{\infty} e^{-i h x} \Phi_{n}(-h) \mathrm{d} h+\int_{0}^{\infty} e^{i h x} \Phi_{n}(h) \mathrm{d} h\right] \\
& \times \int_{0}^{\infty} \cos (x y) f(y) d y \int_{0}^{\infty} \cos (x t) g(t) \mathrm{d} t \\
= & \frac{\sqrt{2}}{\pi \sqrt{\pi}}(-i)^{n} \int_{0}^{\infty} \int_{0}^{\infty} \int_{0}^{\infty}\left(e^{-i h x} \Phi_{n}(-h)+e^{i h x} \Phi_{n}(h)\right) \\
& \times \cos (x y) \cos (x t) f(y) g(t) \mathrm{d} t \mathrm{~d} y \mathrm{~d} h \\
= & \frac{(-i)^{n}}{\pi \sqrt{2 \pi}} \int_{0}^{\infty} \int_{0}^{\infty} \int_{0}^{\infty}\left(e^{-i h x} \Phi_{n}(-h)+e^{i h x} \Phi_{n}(h)\right) \\
& \times(\cos (x(y+t))+\cos (x(y-t))) f(y) g(t) \mathrm{d} t \mathrm{~d} y \mathrm{~d} h \\
= & \frac{(-i)^{n}}{2 \pi \sqrt{2 \pi}} \int_{0}^{\infty} \int_{0}^{\infty} \int_{0}^{\infty}\left(e^{i x(y+t+h)}+e^{-i x(y+t-h)}+e^{i x(y-t+h)}\right. \\
& \left.+e^{-i x(y-t-h)}\right) \Phi_{n}(h) f(y) g(t) \mathrm{d} t \mathrm{~d} y \mathrm{~d} h \\
& +\frac{i^{n}}{2 \pi \sqrt{2 \pi}} \int_{0}^{\infty} \int_{0}^{\infty} \int_{0}^{\infty}\left(e^{i x(y+t-h)}+e^{-i x(y+t+h)}+e^{i x(y-t-h)}\right. \\
& \left.+e^{-i x(y-t+h)}\right) \Phi_{n}(h) f(y) g(t) \mathrm{d} t \mathrm{~d} y \mathrm{~d} h .
\end{aligned}
$$

Thus, if $n=2 k, k \in \mathbb{N}_{0}$, then identity (2.4) and some changes of variables allow to get:

$$
\begin{aligned}
& \Phi_{2 k}(x)\left(T_{c} f\right)(x)\left(T_{c} g\right)(x) \\
& =\frac{(-i)^{2 k}}{\pi \sqrt{2 \pi}} \int_{0}^{\infty} \int_{0}^{\infty} \int_{0}^{\infty}\left(\frac{e^{i x(y+t+h)}+e^{-i x(y+t+h)}}{2}\right. \\
& +\frac{e^{i x(y-t+h)}+e^{-i x(y-t+h)}}{2}+\frac{e^{i x(y-t-h)}+e^{-i x(y-t-h)}}{2} \\
& \left.+\frac{e^{i x(y+t-h)}+e^{-i x(y+t-h)}}{2}\right) \Phi_{2 k}(h) f(y) g(t) \mathrm{d} h \mathrm{~d} y \mathrm{~d} t \\
& =\frac{(-1)^{k}}{\pi \sqrt{2 \pi}} \int_{0}^{\infty} \int_{0}^{\infty} \int_{0}^{\infty}(\cos (x(y+t+h))+\cos (x(y-t+h)) \\
& +\cos (x(y-t-h))+\cos (x(y+t-h))) \Phi_{2 k}(h) f(y) g(t) \mathrm{d} h \mathrm{~d} y \mathrm{~d} t \\
& =\frac{(-1)^{k}}{\pi \sqrt{2 \pi}}\left[\int_{0}^{\infty} \int_{0}^{\infty} \int_{y+t}^{\infty} \cos (x u) \Phi_{2 k}(u-y-t) f(y) g(t) \mathrm{d} u \mathrm{~d} y \mathrm{~d} t\right. \\
& +\int_{0}^{\infty} \int_{0}^{\infty} \int_{y-t}^{\infty} \cos (x u) \Phi_{2 k}(u-y+t) f(y) g(t) \mathrm{d} u \mathrm{~d} y \mathrm{~d} t \\
& +\int_{0}^{\infty} \int_{0}^{\infty} \int_{-\infty}^{y-t} \cos (x u) \Phi_{2 k}(-u+y-t) f(y) g(t) \mathrm{d} u \mathrm{~d} y \mathrm{~d} t \\
& \left.+\int_{0}^{\infty} \int_{0}^{\infty} \int_{-\infty}^{y+t} \cos (x u) \Phi_{2 k}(-u+y+t) f(y) g(t) \mathrm{d} u \mathrm{~d} y \mathrm{~d} t\right] \\
& =\frac{(-1)^{k}}{\pi \sqrt{2 \pi}}\left[\int_{0}^{\infty} \int_{0}^{\infty} \int_{-\infty}^{\infty} \cos (x u) \Phi_{2 k}(u-y-t) f(y) g(t) \mathrm{d} u \mathrm{~d} y \mathrm{~d} t\right. \\
& \left.+\int_{0}^{\infty} \int_{0}^{\infty} \int_{-\infty}^{\infty} \cos (x u) \Phi_{2 k}(u-y+t) f(y) g(t) \mathrm{d} u \mathrm{~d} y \mathrm{~d} t\right]
\end{aligned}
$$




$$
\begin{aligned}
= & \frac{(-1)^{k}}{\pi \sqrt{2 \pi}}\left[\int_{0}^{\infty} \int_{0}^{\infty} \int_{0}^{\infty} \cos (x u) \Phi_{2 k}(u-y-t) f(y) g(t) \mathrm{d} u \mathrm{~d} y \mathrm{~d} t\right. \\
& +\int_{0}^{\infty} \int_{0}^{\infty} \int_{-\infty}^{0} \cos (x u) \Phi_{2 k}(u-y-t) f(y) g(t) \mathrm{d} u \mathrm{~d} y \mathrm{~d} t \\
& +\int_{0}^{\infty} \int_{0}^{\infty} \int_{0}^{\infty} \cos (x u) \Phi_{2 k}(u-y+t) f(y) g(t) \mathrm{d} u \mathrm{~d} y \mathrm{~d} t \\
& \left.+\int_{0}^{\infty} \int_{0}^{\infty} \int_{-\infty}^{0} \cos (x u) \Phi_{2 k}(u-y+t) f(y) g(t) \mathrm{d} u \mathrm{~d} y \mathrm{~d} t\right] \\
= & \sqrt{\frac{2}{\pi}} \int_{0}^{\infty} \cos (x u)\left[\frac { ( - 1 ) ^ { k } } { 2 \pi } \int _ { 0 } ^ { \infty } \int _ { 0 } ^ { \infty } \left(\Phi_{2 k}(u-y-t)+\Phi_{2 k}(-u-y-t)\right.\right. \\
& \left.\left.+\Phi_{2 k}(u-y+t)+\Phi_{2 k}(-u-y+t)\right) f(y) g(t) \mathrm{d} y \mathrm{~d} t\right] \mathrm{d} u \\
= & T_{c}\left(f \circledast \circledast^{e} g\right)(x) .
\end{aligned}
$$

On the other hand, if $n=2 k+1, k \in \mathbb{N}_{0}$, then by applying

$$
2 i \sin z=e^{i z}-e^{-i z}, \quad z \in \mathbb{C},
$$

and a few changes of variables, we have:

$$
\begin{aligned}
& \Phi_{2 k+1}(x)\left(T_{c} f\right)(x)\left(T_{c} g\right)(x) \\
= & \frac{i(-i)^{2 k+1}}{\pi \sqrt{2 \pi}} \int_{0}^{\infty} \int_{0}^{\infty} \int_{0}^{\infty}\left(\frac{e^{i x(y+t+h)}-e^{-i x(y+t+h)}}{2 i}\right. \\
& +\frac{e^{i x(y-t+h)}-e^{-i x(y-t+h)}}{2 i}-\frac{e^{i x(y-t-h)}-e^{-i x(y-t-h)}}{2 i} \\
& \left.-\frac{e^{i x(y+t-h)}-e^{-i x(y+t-h)}}{2 i}\right) \Phi_{2 k+1}(h) f(y) g(t) \mathrm{d} h \mathrm{~d} y \mathrm{~d} t \\
= & \frac{(-i)^{2 k}}{\pi \sqrt{2 \pi}} \int_{0}^{\infty} \int_{0}^{\infty} \int_{0}^{\infty}(\sin (x(y+t+h))+\sin (x(y-t+h)) \\
& -\sin (x(y-t-h))-\sin (x(y+t-h))) \Phi_{2 k+1}(h) f(y) g(t) \mathrm{d} h \mathrm{~d} y \mathrm{~d} t \\
= & \frac{(-1)^{k}}{\pi \sqrt{2 \pi}}\left[\int_{0}^{\infty} \int_{0}^{\infty} \int_{y+t}^{\infty} \sin (x u) \Phi_{2 k+1}(u-y-t) f(y) g(t) \mathrm{d} u \mathrm{~d} y \mathrm{~d} t\right. \\
& +\int_{0}^{\infty} \int_{0}^{\infty} \int_{y-t}^{\infty} \sin (x u) \Phi_{2 k+1}(u-y+t) f(y) g(t) \mathrm{d} u \mathrm{~d} y \mathrm{~d} t \\
& -\int_{0}^{\infty} \int_{0}^{\infty} \int_{-\infty}^{y-t} \sin (x u) \Phi_{2 k+1}(-u+y-t) f(y) g(t) \mathrm{d} u \mathrm{~d} y \mathrm{~d} t \\
& \left.-\int_{0}^{\infty} \int_{0}^{\infty} \int_{-\infty}^{y+t} \sin (x u) \Phi_{2 k+1}(-u+y+t) f(y) g(t) \mathrm{d} u \mathrm{~d} y \mathrm{~d} t\right] \\
= & \frac{(-1)^{k}}{\pi \sqrt{2 \pi}}\left[\int_{0}^{\infty} \int_{0}^{\infty} \int_{-\infty}^{\infty} \sin (x u) \Phi_{2 k+1}(u-y-t) f(y) g(t) \mathrm{d} u \mathrm{~d} y \mathrm{~d} t\right. \\
& \left.+\int_{0}^{\infty} \int_{0}^{\infty} \int_{-\infty}^{\infty} \sin (x u) \Phi_{2 k+1}(u-y+t) f(y) g(t) \mathrm{d} u \mathrm{~d} y \mathrm{~d} t\right] \\
& \sqrt{\frac{2}{\pi}} \int_{0}^{\infty} \sin (x u)\left[\frac { ( - 1 ) ^ { k } } { 2 \pi } \int _ { 0 } ^ { \infty } \int _ { 0 } ^ { \infty } \left(\Phi_{2 k+1}(u-y-t)-\Phi_{2 k+1}(-u-y-t)\right.\right. \\
& \left.\left.+\Phi_{2 k+1}(u-y+t)-\Phi_{2 k+1}(-u-y+t)\right) f(y) g(t) \mathrm{d} y \mathrm{~d} t\right] \mathrm{d} u \\
&
\end{aligned}
$$




$$
=T_{s}\left(f \circledast^{o} g\right)(x) .
$$

We will proceed with additional four new (classes of) convolutions, on the half-line, and their factorization identities.

Definition 2. For any $f$ and $g \in L^{1}\left(\mathbb{R}_{+}\right)$, we define the convolution operators $\odot^{e}$ and $\odot^{o}$ by:

$$
\begin{aligned}
\left(f \odot^{e} g\right)(u) & =\frac{(-1)^{k}}{2 \pi} \int_{0}^{\infty} \int_{0}^{\infty}\left[\Phi_{2 k}(y-t-u)+\Phi_{2 k}(y-t+u)\right. \\
& \left.-\Phi_{2 k}(y+t-u)-\Phi_{2 k}(y+t+u)\right] f(y) g(t) \mathrm{d} y \mathrm{~d} t, \\
\left(f \odot^{o} g\right)(u) & =\frac{(-1)^{k}}{2 \pi} \int_{0}^{\infty} \int_{0}^{\infty}\left[\Phi_{2 k+1}(-y+t+u)-\Phi_{2 k+1}(-y+t-u)\right. \\
& \left.+\Phi_{2 k+1}(y+t-u)-\Phi_{2 k+1}(y+t+u)\right] f(y) g(t) \mathrm{d} y \mathrm{~d} t,
\end{aligned}
$$

$k \in \mathbb{N}_{0}$.

Theorem 2. Let $f, g \in L^{1}\left(\mathbb{R}_{+}\right)$. The convolutions $\odot^{e}$ and $\odot^{o}$ of functions $f$ and $g$ belong to $L^{1}\left(\mathbb{R}_{+}\right)$and satisfy the following weighted factorization identities associated with cosine and sine Fourier integral transforms $T_{c}$ and $T_{s}$ :

$$
\begin{aligned}
& T_{c}\left(f \odot^{e} g\right)(x)=\Phi_{2 k}(x)\left(T_{s} f\right)(x)\left(T_{s} g\right)(x), \\
& T_{s}\left(f \odot^{o} g\right)(x)=\Phi_{2 k+1}(x)\left(T_{s} f\right)(x)\left(T_{s} g\right)(x), \quad k \in \mathbb{N}_{0} .
\end{aligned}
$$

Proof. This proof is performed with the same technique already presented in the proof of Theorem 1, by using the identities (1.1), the trigonometric formulas $2 \sin \alpha \sin \beta=\cos (\alpha-\beta)-$ $\cos (\alpha+\beta), 2 \cos z=e^{i z}+e^{-i z}$ and $2 i \sin z=e^{i z}-e^{-i z}(\alpha, \beta \in \mathbb{R}, z \in \mathbb{C})$, the property (1.2), the oddness of the function $\sin (x)$ and the evenness of $\cos (x)$. In view of this, a detailed deduction is here omitted.

Indeed, from inequalities:

$$
\int_{0}^{\infty}\left|\left(f \odot^{e} g\right)(u)\right| \mathrm{d} u \leq \frac{2}{\pi} \int_{0}^{\infty}|f(t)| \mathrm{d} t \times \int_{0}^{\infty}|g(t)| \mathrm{d} t \times \int_{0}^{\infty}\left|\Phi_{2 k}(t)\right| \mathrm{d} t<\infty,
$$

and

$$
\int_{0}^{\infty}\left|\left(f \odot^{o} g\right)(u)\right| d u \leq \frac{2}{\pi} \int_{0}^{\infty}|f(t)| \mathrm{d} t \times \int_{0}^{\infty}|g(t)| \mathrm{d} t \times \int_{0}^{\infty}\left|\Phi_{2 k+1}(t)\right| \mathrm{d} t<\infty
$$

we conclude that $f \odot^{e} g, \quad f \odot^{o} g \in L^{1}\left(\mathbb{R}_{+}\right)$for $f, g \in L^{1}\left(\mathbb{R}_{+}\right)$. Moreover:

$$
\begin{aligned}
& \Phi_{n}(x)\left(T_{s} f\right)(x)\left(T_{s} g\right)(x) \\
= & \frac{(-i)^{n}}{2 \pi \sqrt{2 \pi}}(-1)^{n} \int_{0}^{\infty} \int_{0}^{\infty} \int_{0}^{\infty}\left(e^{i x(y-t-h)}+e^{-i x(y-t+h)}-e^{i x(y+t-h)}\right. \\
& \left.-e^{-i x(y+t+h)}\right) \Phi_{n}(h) f(y) g(t) \mathrm{d} t \mathrm{~d} y \mathrm{~d} h \\
& +\frac{(-i)^{n}}{2 \pi \sqrt{2 \pi}} \int_{0}^{\infty} \int_{0}^{\infty} \int_{0}^{\infty}\left(e^{i x(y-t+h)}+e^{-i x(y-t-h)}-e^{i x(y+t+h)}\right. \\
& \left.-e^{-i x(y+t-h)}\right) \Phi_{n}(h) f(y) g(t) \mathrm{d} t \mathrm{~d} y \mathrm{~d} h .
\end{aligned}
$$


Thus, if $n=2 k, k \in \mathbb{N}_{0}$, we have:

$$
\begin{aligned}
& \Phi_{2 k}(x)\left(T_{s} f\right)(x)\left(T_{s} g\right)(x) \\
= & \frac{i^{2 k}}{\pi \sqrt{2 \pi}} \int_{0}^{\infty} \int_{0}^{\infty} \int_{0}^{\infty}(\cos (x(y-t-h))-\cos (x(y+t-h)) \\
& +\cos (x(y-t+h))-\cos (x(y+t+h))) \Phi_{2 k}(h) f(y) g(t) \mathrm{d} t \mathrm{~d} y \mathrm{~d} h \\
= & \sqrt{\frac{2}{\pi}} \int_{0}^{\infty} \cos (x u)\left[\frac { i ^ { 2 k } } { 2 \pi } \int _ { 0 } ^ { \infty } \int _ { 0 } ^ { \infty } \left(\Phi_{2 k}(y-t-u)+\Phi_{2 k}(y-t+u)\right.\right. \\
& \left.\left.-\Phi_{2 k}(y+t-u)-\Phi_{2 k}(y+t+u)\right) f(y) g(t) \mathrm{d} t \mathrm{~d} y\right] \mathrm{d} u \\
= & T_{c}\left(f \odot^{e} g\right)(x) .
\end{aligned}
$$

For the case $n=2 k+1, k \in \mathbb{N}_{0}$, we obtain:

$$
\begin{aligned}
& \Phi_{2 k+1}(x)\left(T_{s} f\right)(x)\left(T_{s} g\right)(x) \\
= & \frac{(-i)^{2 k}}{\pi \sqrt{2 \pi}} \int_{0}^{\infty} \int_{0}^{\infty} \int_{0}^{\infty}(\sin (x(y-t+h))-\sin (x(y-t-h)) \\
& +\sin (x(y+t-h))-\sin (x(y+t+h)) \Phi_{2 k+1}(h) f(y) g(t) \mathrm{d} t \mathrm{~d} y \mathrm{~d} h, \\
= & \sqrt{\frac{2}{\pi}} \int_{0}^{\infty} \sin (x u)\left[\frac { i ^ { 2 k } } { 2 \pi } \int _ { 0 } ^ { \infty } \int _ { 0 } ^ { \infty } \left(\Phi_{2 k+1}(-y+t+u)\right.\right. \\
& -\Phi_{2 k+1}(-y+t-u)+\Phi_{2 k+1}(y+t-u) \\
& \left.\left.-\Phi_{2 k+1}(y+t+u)\right) f(y) g(t) \mathrm{d} t \mathrm{~d} y\right] \mathrm{d} u \\
= & T_{s}\left(f \odot^{o} g\right)(x) .
\end{aligned}
$$

Definition 3. For any $f$ and $g \in L^{1}\left(\mathbb{R}_{+}\right)$, we define the convolution operators $\oplus^{e}$ and $\oplus^{o}$ by:

$$
\begin{aligned}
\left(f \oplus^{e} g\right)(u) & =\frac{(-1)^{k}}{2 \pi} \int_{0}^{\infty} \int_{0}^{\infty}\left[\Phi_{2 k}(y+t-u)-\Phi_{2 k}(y+t+u)\right. \\
& \left.+\Phi_{2 k}(y-t-u)-\Phi_{2 k}(y-t+u)\right] f(y) g(t) \mathrm{d} y \mathrm{~d} t \\
\left(f \oplus^{o} g\right)(u) & =\frac{(-1)^{k}}{2 \pi} \int_{0}^{\infty} \int_{0}^{\infty}\left[\Phi_{2 k+1}(y+t-u)+\Phi_{2 k+1}(y+t+u)\right. \\
& \left.+\Phi_{2 k+1}(y-t-u)+\Phi_{2 k+1}(y-t+u)\right] f(y) g(t) \mathrm{d} y \mathrm{~d} t,
\end{aligned}
$$

$k \in \mathbb{N}_{0}$.

Theorem 3. Let $f, g \in L^{1}\left(\mathbb{R}_{+}\right)$. The convolutions $\oplus^{e}$ and $\oplus^{o}$ of $f$ and $g$ belong to $L^{1}\left(\mathbb{R}_{+}\right)$and satisfy the following weighted factorization identities associated with cosine and sine Fourier integral transforms $T_{c}$ and $T_{s}$ :

$$
\begin{aligned}
& T_{s}\left(f \oplus^{e} g\right)(x)=\Phi_{2 k}(x)\left(T_{s} f\right)(x)\left(T_{c} g\right)(x), \\
& T_{c}\left(f \oplus^{o} g\right)(x)=\Phi_{2 k+1}(x)\left(T_{s} f\right)(x)\left(T_{c} g\right)(x), \quad k \in \mathbb{N}_{0} .
\end{aligned}
$$

Proof. The result is deduced using the technique already presented in detail in the proof of Theorem 1, and considering in this case the identities (1.1), the trigonometric formulas 
$2 \sin \alpha \cos \beta=\sin (\alpha-\beta)+\sin (\alpha+\beta), 2 \cos z=e^{i z}+e^{-i z}$ and $2 i \sin z=e^{i z}-e^{-i z}(\alpha, \beta \in \mathbb{R}$, $z \in \mathbb{C}$ ), the property $(1.2)$, the oddness of the function $\sin (x)$, and the evenness of $\cos (x)$.

Proceeding in the same way as in the proof of Theorems 1 and 2, we obtain that $f \oplus^{e}$ $g, \quad f \oplus^{o} g \in L^{1}\left(\mathbb{R}_{+}\right)$.

As about the factorization identities, we have:

$$
\begin{aligned}
& \Phi_{n}(x)\left(T_{s} f\right)(x)\left(T_{c} g\right)(x) \\
= & \frac{(-i)^{n}}{2 \pi i \sqrt{2 \pi}} \int_{0}^{\infty} \int_{0}^{\infty} \int_{0}^{\infty} \Phi_{n}(-h)\left[e^{i x(y+t-h)}-e^{-i x(y+t+h)}+e^{i x(y-t-h)}\right. \\
& \left.-e^{-i x(y-t+h)}\right] f(y) g(t) \mathrm{d} y \mathrm{~d} t \mathrm{~d} h \\
& +\frac{(-i)^{n}}{2 \pi i \sqrt{2 \pi}} \int_{0}^{\infty} \int_{0}^{\infty} \int_{0}^{\infty} \Phi_{n}(h)\left[e^{i x(y+t+h)}-e^{-i x(y+t-h)}+e^{i x(y-t+h)}\right. \\
& \left.-e^{-i x(y-t-h)}\right] f(y) g(t) \mathrm{d} y \mathrm{~d} t \mathrm{~d} h .
\end{aligned}
$$

Thus, if $n=2 k, k \in \mathbb{N}_{0}$, we have:

$$
\begin{aligned}
& \Phi_{2 k}(x)\left(T_{s} f\right)(x)\left(T_{c} g\right)(x) \\
= & \frac{i^{2 k}}{\pi \sqrt{2 \pi}} \int_{0}^{\infty} \int_{0}^{\infty} \int_{0}^{\infty} \Phi_{2 k}(h)[\sin (x(y+t-h))+\sin (x(y+t+h)) \\
& +\sin (x(y-t-h))+\sin (x(y-t+h))] f(y) g(t) \mathrm{d} y \mathrm{~d} t \mathrm{~d} h \\
= & \sqrt{\frac{2}{\pi}} \int_{0}^{\infty} \sin (x u)\left[\frac { i ^ { 2 k } } { 2 \pi } \int _ { 0 } ^ { \infty } \int _ { 0 } ^ { \infty } \left(\Phi_{2 k}(y+t-u)-\Phi_{2 k}(y+t+u)\right.\right. \\
& \left.\left.+\Phi_{2 k}(y-t-u)-\Phi_{2 k}(y-t+u)\right) f(y) g(t) \mathrm{d} y \mathrm{~d} t\right] \mathrm{d} u \\
= & T_{s}\left(f \oplus^{e} g\right)(x) .
\end{aligned}
$$

On the other hand, if $n=2 k+1, k \in \mathbb{N}_{0}$, then:

$$
\begin{aligned}
& \Phi_{2 k+1}(x)\left(T_{s} f\right)(x)\left(T_{c} g\right)(x) \\
= & \frac{i^{2 k}}{\pi \sqrt{2 \pi}} \int_{0}^{\infty} \int_{0}^{\infty} \int_{0}^{\infty} \Phi_{2 k+1}(h)[-\cos (x(y+t+h)) \\
& +\cos (x(y+t-h))-\cos (x(y-t+h))+\cos (x(y-t-h))] \\
& \times f(y) g(t) \mathrm{d} y \mathrm{~d} t \mathrm{~d} h \\
= & \sqrt{\frac{2}{\pi}} \int_{0}^{\infty} \cos (x u)\left[\frac { i ^ { 2 k } } { 2 \pi } \int _ { 0 } ^ { \infty } \int _ { 0 } ^ { \infty } \left(\Phi_{2 k+1}(y+t-u)\right.\right. \\
& +\Phi_{2 k+1}(y+t+u)+\Phi_{2 k+1}(y-t-u) \\
& \left.\left.+\Phi_{2 k+1}(y-t+u)\right) f(y) g(t) \mathrm{d} y \mathrm{~d} t\right] \mathrm{d} u \\
= & T_{c}\left(f \oplus^{o} g\right)(x) .
\end{aligned}
$$

\section{Applications to Integral Equations}

Our aim in this section is to study certain integral equations (and systems of integral equations) generated by the previously presented convolutions, to establish the solvability of such convolution integral equations and to obtain consequent explicit solution formulas. 


\subsection{System of Integral Equations}

We start by analysing a system of integral equations involving two of the previously introduced convolutions. Indeed, let us consider the following system of integral equations:

$$
\left\{\begin{array}{l}
\varphi(x)+\lambda_{1}\left(q \circledast^{o} \psi\right)(x)=f(x) \\
\psi(x)+\lambda_{2}\left(p \odot^{e} \varphi\right)(x)=g(x)
\end{array}, \quad x \in \mathbb{R}_{+},\right.
$$

where $\varphi$ and $\psi$ are the unknown functions, $p, q, f$ and $g \in L^{1}\left(\mathbb{R}_{+}\right)$are given functions, and $\lambda_{1}$ and $\lambda_{2}$ denote complex numbers.

Applying the Fourier sine to the first equation of (3.5) and Fourier cosine to the second one, we obtain the following linear system of algebraic equations:

$$
\left\{\begin{array}{c}
T_{s}(\varphi)+\lambda_{1} \Phi_{2 k+1} T_{c}(q) T_{c}(\psi)=T_{s}(f) \\
T_{c}(\psi)+\lambda_{2} \Phi_{2 k} T_{s}(p) T_{s}(\varphi)=T_{c}(g) .
\end{array}\right.
$$

Suppose that:

$$
1-\lambda_{1} \lambda_{2} \Phi_{2 k} \Phi_{2 k+1} T_{s}(p) T_{c}(q) \neq 0 .
$$

Then, the linear system (3.6) has the solution:

$$
\begin{aligned}
T_{s}(\varphi) & =\frac{T_{s}(f)-\lambda_{1} \Phi_{2 k+1} T_{c}(q) T_{c}(g)}{1-\lambda_{1} \lambda_{2} \Phi_{2 k} \Phi_{2 k+1} T_{s}(p) T_{c}(q)} \\
T_{c}(\psi) & =\frac{T_{c}(g)-\lambda_{2} \Phi_{2 k} T_{s}(p) T_{s}(f)}{1-\lambda_{1} \lambda_{2} \Phi_{2 k} \Phi_{2 k+1} T_{s}(p) T_{c}(q)} .
\end{aligned}
$$

Consider the function:

$$
H(t)=\frac{\lambda_{1} \lambda_{2} \Phi_{2 k} t}{1-\lambda_{1} \lambda_{2} \Phi_{2 k} t}
$$

where

$$
t=T_{c}\left(p \oplus^{o} q\right)=\Phi_{2 k+1} T_{s}(p) T_{c}(q) .
$$

Since $t:=t(x)$ is the cosine Fourier transform of a function of $L^{1}\left(\mathbb{R}_{+}\right)$, and $H(t)$ is analytic under the condition (3.7) and $H(0)=0$, then by the Wiener-Lévy theorem (cf. [7, p. 63]), there exists a function $h \in L^{1}\left(\mathbb{R}_{+}\right)$such that:

$$
T_{c}(h)=H(t)=\frac{\lambda_{1} \lambda_{2} \Phi_{2 k} \Phi_{2 k+1} T_{s}(p) T_{c}(q)}{1-\lambda_{1} \lambda_{2} \Phi_{2 k} \Phi_{2 k+1} T_{s}(p) T_{c}(q)} .
$$

(Note that, for Fourier cosine transform, the Wiener-Lévy Theorem states that if $\tau(x)$ is a Fourier cosine transform of an $L^{1}\left(\mathbb{R}_{+}\right)$function and $\Phi(u)$ is analytic in the neighborhood of the origin with $\Phi(0)=0$, over the range of values of $\tau(x)$, then $\Phi(\tau(x))$ is also the Fourier cosine transform of an $L^{1}\left(\mathbb{R}_{+}\right)$function.)

Thus, we obtain:

$$
\begin{aligned}
T_{s}(\varphi)= & {\left[T_{s}(f)-\lambda_{1} \Phi_{2 k+1} T_{c}(q) T_{c}(g)\right]\left[1+T_{c}(h)\right] } \\
= & T_{s}(f)-\lambda_{1} \Phi_{2 k+1} T_{c}(q) T_{c}(g)+T_{s}(f) T_{c}(h) \\
& -\lambda_{1} \Phi_{2 k+1} T_{c}(q) T_{c}(g) T_{c}(h) \\
= & T_{s}(f)-\lambda_{1} T_{s}\left(q \circledast^{o} g\right)+T_{s}\left(f *^{1} h\right)-\lambda_{1} T_{s}\left(\left(q \circledast^{o} g\right) *^{1} h\right) .
\end{aligned}
$$


Hence:

$$
\varphi=f+f *^{1} h-\lambda_{1} q \circledast^{o} g-\lambda_{1}\left(q \circledast{ }^{o} g\right) *^{1} h .
$$

Similarly, we have:

$$
\begin{aligned}
T_{c}(\psi)= & {\left[T_{c}(g)-\lambda_{2} \Phi_{2 k} T_{s}(p) T_{s}(f)\right]\left[1+T_{c}(h)\right] } \\
= & T_{c}(g)+T_{c}(g) T_{c}(h)-\lambda_{2} \Phi_{2 k} T_{s}(p) T_{s}(f) \\
& -\lambda_{2} \Phi_{2 k} T_{s}(p) T_{s}(f) T_{c}(h) \\
= & T_{c}(g)+T_{c}\left(g *^{0} h\right)-\lambda_{2} T_{c}\left(p \odot^{e} f\right)-\lambda_{2} T_{c}\left(\left(p \odot^{e} f\right) *^{0} h\right) .
\end{aligned}
$$

Thus:

$$
\psi=g+g *^{0} h-\lambda_{2} p \odot^{e} f-\lambda_{2}\left(p \odot^{e} f\right) *^{0} h .
$$

\subsection{Convolution Equations}

Let us now consider the following (independent) integral equations in $L^{1}\left(\mathbb{R}_{+}\right)$:

$$
\begin{aligned}
& \lambda_{1} \varphi(x)+\left(p \circledast \circledast^{e} \varphi\right)(x)=f(x), \\
& \lambda_{2} \psi(x)+\left(q \odot^{o} \psi\right)(x)=g(x),
\end{aligned}
$$

where $\lambda_{1}, \lambda_{2} \in \mathbb{C}, p, q, f$ and $g \in L^{1}\left(\mathbb{R}_{+}\right)$are given and $\varphi$ and $\psi$ are unknown elements to be determined in the same space. Let us fix the notation

$$
\begin{aligned}
& A(x):=\lambda_{1}+\Phi_{2 k}(x)\left(T_{c} p\right)(x), \\
& B(x):=\lambda_{2}+\Phi_{2 k+1}(x)\left(T_{s} q\right)(x) .
\end{aligned}
$$

Theorem 4. Assume that $A(x) \neq 0, B(x) \neq 0$, for $x \in \mathbb{R}_{+}$, and $\frac{T_{c} f}{A}, \frac{T_{s} g}{B} \in L^{1}\left(\mathbb{R}_{+}\right)$. Then: (i) Eq. (3.8) has a solution in $L^{1}\left(\mathbb{R}_{+}\right)$if and only if:

$$
T_{c}\left(\frac{T_{c} f}{A}\right) \in L^{1}\left(\mathbb{R}_{+}\right) ;
$$

(ii) Eq. (3.9) has a solution in $L^{1}\left(\mathbb{R}_{+}\right)$if and only if:

$$
T_{s}\left(\frac{T_{s} g}{B}\right) \in L^{1}\left(\mathbb{R}_{+}\right) .
$$

In these cases (3.10) and (3.11), the solutions of Eqs. (3.8) and (3.9) are given, respectively, by:

$$
\varphi=T_{c}\left(\frac{T_{c} f}{A}\right), \quad \psi=T_{s}\left(\frac{T_{s} g}{B}\right)
$$

Proof. Let us prove proposition (i). Suppose that Eq. (3.8) has a solution $\varphi \in L^{1}\left(\mathbb{R}_{+}\right)$. Applying the Fourier cosine $T_{c}$ to both sides of Eq. (3.8), we obtain:

$$
\lambda_{1}\left(T_{c} \varphi\right)(x)+\Phi_{2 k}(x)\left(T_{c} p\right)(x)\left(T_{c} \varphi\right)(x)=\left(T_{c} f\right)(x),
$$


that is:

$$
A(x)\left(T_{c} \varphi\right)(x)=\left(T_{c} f\right)(x) .
$$

Having in mind that $A(x) \neq 0$, for $x \in \mathbb{R}_{+}$, we get:

$$
\left(T_{c} \varphi\right)(x)=\frac{\left(T_{c} f\right)(x)}{A(x)}
$$

Moreover, since $\frac{T_{c} f}{A} \in L^{1}\left(\mathbb{R}_{+}\right)$, we obtain:

$$
\varphi=T_{c}\left(\frac{T_{c} f}{A}\right) \in L^{1}\left(\mathbb{R}_{+}\right)
$$

Assume now that $\varphi=T_{c}\left(\frac{T_{c} f}{A}\right) \in L^{1}\left(\mathbb{R}_{+}\right)$. Thus, we get:

$$
T_{c}\left(\lambda_{1} \varphi(x)+\left(p \circledast \circledast^{e} \varphi\right)(x)\right)=\left(T_{c} f\right)(x) .
$$

Thus, applying the inverse of Fourier cosine transform to both members of the last equation, we conclude that $\varphi$ fulfills Eq. (3.8).

To consider case $(i i)$, establish the solvability of (3.9) and obtain its solution, we proceed in the same way, making use of the Fourier sine transform $T_{s}$.

\section{Acknowledgments}

This work was supported by Fundação para a Ciência e a Tecnologia (FCT), within project UIDB/04106/2020 (CIDMA) and by national funds (OE), through FCT, I.P., in the scope of the framework contract foreseen in the numbers 4, 5 and 6 of the article 23, of the Decree-Law 57/2016, of August 29, changed by Law 57/2017, of July 19.

The authors gratefully thank the Referee for the constructive comments and recommendations which definitely helped improving the readability and quality of the paper.

\section{References}

[1] Anh, P.K., Castro, L.P., Thao, P.T., Tuan, N.M.: Two new convolutions for the fractional Fourier transform. Wireless Pers. Commun., 92(2), 623-637 (2017)

[2] Castro, L.P., Guerra, R.C., Tuan, N.M.: On Wiener's Tauberian theorems and convolution for oscillatory integral operators. Turk. J. Math., 15(3), 1124-1147 (2019)

[3] Castro, L.P., Guerra, R.C., Tuan, N.M.: Convolution theorems related with the solvability of Wiener-Hopf plus Hankel integral equations and Shannon's sampling formula. Math. Slovaca, 69(5), 1149-1164 (2019)

[4] Castro, L.P., Minh, L.T., Tuan, N.M.: New convolutions for quadratic-phase Fourier integral operators and their applications. Mediterr. J. of Math., 15(13), 17pp. (2018)

[5] Castro, L.P., Saitoh, S.: New convolutions and norm inequalities. Math. Inequal. Appl., 15(3), 707-716 (2012) 
[6] Duduchava, R.: Integral Equations in Convolution with Discontinuous Presymbols. Singular Integral Equations with Fixed Singularities, and Their Applications to Some Problems of Mechanics. Teubner-Texte zur Mathematik. BSB B.G. Teubner Verlagsgesellschaft, Leipzig (1979).

[7] Paley, R., Wiener, N.: Fourier Transforms in the Complex Domain. AMS Coll. Publ. XIX, New York (1934)

[8] Sneddon, I.N.: Fourier Transforms. McGray-Hill, New York (1951)

[9] Titchmarsh, E.C.: Introduction to the Theory of Fourier Integrals. Oxford University Press, London (1948). 